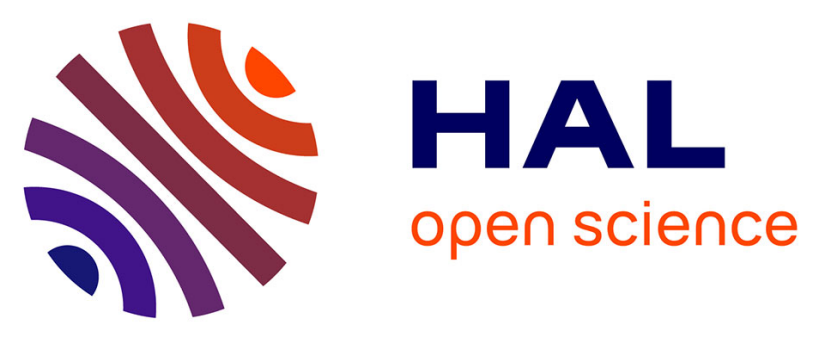

\title{
HgSe self-doped nanocrystals as a platform to investigate the effects of vanishing confinement
}

Bertille Martinez, Clément Livache, L.Donald Mouafo Notemgnou, Nicolas

Goubet, Sean Keuleyan, Hervé Cruguel, Sandrine Ithurria, Hervé Aubin, Abdelkarim Ouerghi, Bernard Doudin, et al.

\section{To cite this version:}

Bertille Martinez, Clément Livache, L.Donald Mouafo Notemgnou, Nicolas Goubet, Sean Keuleyan, et al.. HgSe self-doped nanocrystals as a platform to investigate the effects of vanishing confinement. ACS Applied Materials \& Interfaces, 2017, 9 (41), pp.36173-36180. 10.1021/acsami.7b10665 . hal01597949

\section{HAL Id: hal-01597949 \\ https://hal.science/hal-01597949}

Submitted on 15 Nov 2017

HAL is a multi-disciplinary open access archive for the deposit and dissemination of scientific research documents, whether they are published or not. The documents may come from teaching and research institutions in France or abroad, or from public or private research centers.
L'archive ouverte pluridisciplinaire HAL, est destinée au dépôt et à la diffusion de documents scientifiques de niveau recherche, publiés ou non, émanant des établissements d'enseignement et de recherche français ou étrangers, des laboratoires publics ou privés. 
Subscriber access provided by BUPMC - Bibliothèque Universitaire Pierre et Marie Curie

Article

\section{HgSe self-doped nanocrystals as a platform to investigate the effects of vanishing confinement}

Bertille Martinez, Clément Livache, L.Donald Mouafo Notemgnou, Nicolas Goubet, Sean Keuleyan, Herve Cruguel, Sandrine Ithurria, Herve Aubin, Abdelkarim Ouerghi, Bernard Doudin, Emmanuelle Lacaze, Benoit Dubertret, Mathieu G. Silly, Ricardo Psm Lobo, Jean Francois Dayen, and Emmanuel Lhuillier

ACS Appl. Mater. Interfaces, Just Accepted Manuscript • DOI: 10.1021/acsami.7b10665 • Publication Date (Web): 28 Sep 2017

Downloaded from http://pubs.acs.org on September 29, 2017

\section{Just Accepted}

"Just Accepted" manuscripts have been peer-reviewed and accepted for publication. They are posted online prior to technical editing, formatting for publication and author proofing. The American Chemical Society provides "Just Accepted" as a free service to the research community to expedite the dissemination of scientific material as soon as possible after acceptance. "Just Accepted" manuscripts appear in full in PDF format accompanied by an HTML abstract. "Just Accepted" manuscripts have been fully peer reviewed, but should not be considered the official version of record. They are accessible to all readers and citable by the Digital Object Identifier (DOI®). "Just Accepted" is an optional service offered to authors. Therefore, the "Just Accepted" Web site may not include all articles that will be published in the journal. After a manuscript is technically edited and formatted, it will be removed from the "Just Accepted" Web site and published as an ASAP article. Note that technical editing may introduce minor changes to the manuscript text and/or graphics which could affect content, and all legal disclaimers and ethical guidelines that apply to the journal pertain. ACS cannot be held responsible for errors or consequences arising from the use of information contained in these "Just Accepted" manuscripts. 


\title{
HgSe self-doped nanocrystals as a platform to investigate the effects of vanishing confinement
}

Bertille Martinez ${ }^{1,2}$, Clément Livache ${ }^{1,2}$, L. Donald Mouafo Notemgnou ${ }^{3}$, Nicolas Goubet $^{1,2}$, Sean Keuleyan ${ }^{4}$, Hervé Cruguel ${ }^{1}$, Sandrine Ithurria ${ }^{2,2 b}$, Hervé Aubin ${ }^{2,2 b}$, Abdelkarim Ouerghi $^{5}$, Bernard Doudin ${ }^{3}$, Emmanuelle Lacaze $^{1}$, Benoit Dubertret ${ }^{2,2 b}$, Mathieu G. Silly ${ }^{6}$, Ricardo P.S.M. Lobo ${ }^{2,2 b}$, Jean Francois Dayen ${ }^{3}$, Emmanuel Lhuillier $^{1^{*}}$

${ }^{1}$ Sorbonne Universités, UPMC Univ. Paris 06, CNRS-UMR 7588, Institut des NanoSciences de Paris, 4 place Jussieu, 75005 Paris, France

${ }^{2}$ LPEM, ESPCI Paris, PSL Research University, 10 rue Vauquelin, 75005 Paris France

${ }^{2 b}$ Sorbonne Universités, UPMC Univ Paris 06, LPEM, 75005 Paris France

${ }^{3}$ Université de Strasbourg, IPCMS-CMRS UMR 7504, 23 Rue du Loess, 67034 Strasbourg, France

${ }^{4}$ Voxtel, Inc., University of Oregon, CAMCOR, 1241 University of Oregon, Eugene, OR 97403, USA

${ }^{5}$ Centre de Nanosciences et de Nanotechnologies, CNRS, Univ. Paris-Sud, Université Paris-Saclay, C2N Marcoussis, 91460 Marcoussis, France

${ }^{6}$ Synchrotron-SOLEIL, Saint-Aubin, BP48, F91192 Gif sur Yvette Cedex, France

\begin{abstract}
Self-doped colloidal quantum dots (CQDs) attract a strong interest for the design of a new generation of low cost infrared optoelectronic devices because of the tunable intraband absorption feature in the mid IR. However, very little remains known on their electronic structure which combines confinement and an inverted band structure, complicating the design of optimized devices. We use a combination of IR spectroscopy and photoemission to determine the absolute energy levels of HgSe CQDs with various sizes and surface chemistries. We demonstrate that the filling of the CQD states ranges from 2 electrons per CQD at small size $(<5 \mathrm{~nm})$ to above 18 electrons per CQD at large sizes $(\approx 20 \mathrm{~nm})$. HgSe CQDs are also an interesting platform to observe vanishing confinement in colloidal nanoparticles. We present evidences for a semiconductor-to-metal transition at the CQD level, through temperature dependent absorption and transport measurements. In contrast with the bulk systems, the transition is the result of the vanishing confinement rather than the increase of the doping level.
\end{abstract}

Keywords: nanocrystals, photoemission, self-doping, semiconductor to metal transition, HgSe, infrared.

*To whom correspondence should be sent: el@insp.upmc.fr 


\section{INTRODUCTION}

Thanks to their widely tunable optical features from UV to $\mathrm{THz}^{1}$, colloidal quantum dots (CQD) are generating significant interest for the design of optoelectronic devices ${ }^{2,3}$. Among the possible applications, low cost alternatives to epitaxially grown semiconductors (InSb, HgCdTe, GaAs/AIGAs and InAs/GaSb...) for mid infrared (IR) photodetectors is attracting a lot of research efforts ${ }^{4,5}$. References 6 and 7 give recent reviews on the field. In the mid IR range, mercury chalcogenides $(\mathrm{HgX})$ have become the most investigated materials because of their strong absorption and photoconductivity ${ }^{8-10}$. While HgTe CQDs appear nearly intrinsic, $\mathrm{HgS}^{11,12}$ and $\mathrm{HgSe}^{1,13}$ are self-doped nanocrystals, presenting excess electrons after their synthesis. As a result, their absorption spectrum is characterized by an additional feature in the mid-IR, resulting from transitions within the conduction band. The possibility to use this intraband feature opens new directions for the design of complex mid-IR emitting and absorbing heterostructures. The intraband can be tuned through the mid-IR spectrum up to even the long-wavelength IR with moderate CQD sizes.

However, the design of optimized devices is currently hampered by the lack of knowledge of the electronic structure of these materials. $\mathrm{HgTe}$ and $\mathrm{HgSe}$ CQDs combine an inverted band structure ${ }^{14,15}$ with quantum confinement, and their spectrum also strongly depends on temperature and surface chemistry ${ }^{16,17}$. More experimental data is consequently needed for the design of optimal ohmic contacts with efficient dissociation of the photogenerated electron hole pairs. Such an effort has been conducted for PbS CQDs with the design of improved solar cells ${ }^{18-20}$. This insight, however, is largely lacking for $\mathrm{HgX}$ CQDs and more needs to be done to determine their absolute energy levels (i.e. with respect to vacuum) and down to the single particle scale ${ }^{21}$. Only recently the Guyot-Sionnest's group reported a systematic investigation of the electronic structure of $\mathrm{HgXCQDs^{22 }}$ based on electrochemistry. However, the effect of surface chemistry remains unknown for $\mathrm{HgX} C \mathrm{CQD}$ while it has been demonstrated to be of utmost importance for other materials ${ }^{18,20}$.

Applications aside (i.e. design of optimized devices), investigation of the electronic structure of self-doped HgSe CQDs is also driven by their vanishing confinement. As a result, HgSe CQDs are an interesting platform to investigate the transition between a confined semiconductor to a metallic system. It has been recently observed in doped quantum wells ${ }^{23}$, and self-doped CQDs ${ }^{17}$ that the optical properties switch from an intraband peak to a plasmon resonance when the confining size is increased. More evidence for this phase transition must now be provided, together with an exploration of its impact on CQD film charge transport properties.

According to the Mott criterion, a material will show metallic character if the product of the Bohr radius $\left(a_{0}\right)$ and the cube root of the carrier density $(n)$ exceeds a threshold value $a_{0} n^{1 / 3}>0.25 .{ }^{24,25}$. Above the threshold doping density, the population of free carriers will be independent of temperature. In HgSe, the dielectric constant $\left(\varepsilon_{r} \approx 16\right)$ is quite large and the Bohr radius given by $a_{0}=\frac{h^{2} \varepsilon_{0} \varepsilon_{r}}{\pi m^{*} e^{2}}$ is around $17 \mathrm{~nm}$, with $h$ the Planck constant, $\varepsilon_{0}$ the vacuum permittivity, $m^{*}$ the electron effective mass $\left(m^{*}=0.05 m_{0}\right.$ with $m_{0}$ the free electron mass) and $e$ the proton charge. The insulator to metal transition is thus expected to occur near $\mathrm{n}=3 \times 10^{15} \mathrm{~cm}^{-3}$; far below the carrier concentration in a HgSe CQD film $\left(n_{\text {film }} \approx 2.6 \times 10^{19} \mathrm{~cm}^{-3}\right.$, assuming two carriers per CQD in a film of randomly close packed CQD with a $4.5 \mathrm{~nm}$ size). In doped quantum wells, it was pointed out that confinement can increase the Mott threshold doping level. ${ }^{26}$ However, metallic character should be observable in large HgSe CQDs only where the confinement is significantly relaxed.

We use a combination of IR spectroscopy and X-ray photoemission spectroscopy (XPS ) to determine the electronic structure of HgSe CQD as a function of size and capping ligands on an absolute energy scale. We show that as we switch from small to large CQDs, the electron population increases from 2, to above 18 per CQD. Using photoemission we demonstrate the presence of surface states resulting from the 
hybridization of $\mathrm{Hg}$ atoms with the ligands. This state is responsible for the surface tunable doping observed in HgX self-doped CQD. We then investigate the temperature dependence of the absorption and conductance of the HgSe film for different sizes of CQDs and conclude that individual CQDs switch from semiconductor behavior, driven by single electron physics, to metallic character, where collective effects emerge. We finally rationalize the result by proposing a phase diagram which includes both the effects of confinement and surface chemistry.

\section{EXPERIMENTAL SECTION}

\section{Nanocrystal synthesis}

Small HgSe CQDs: In a $50 \mathrm{~mL}$ three neck flask, $0.5 \mathrm{~g}$ of mercury acetate $\left(\mathrm{Hg}(\mathrm{OAc})_{2}\right)+10 \mathrm{~mL}$ of oleic acid $(\mathrm{OA})+25$ $\mathrm{mL}$ of oleylamine (OLA) are degassed at $85^{\circ} \mathrm{C}$ for $1 \mathrm{~h}$. Under $\mathrm{Ar}$ at $80^{\circ} \mathrm{C}, 1.6 \mathrm{~mL}$ of a $1 \mathrm{M}$ solution of Selenium (Se) in trioctylphosphine (TOP) is quickly injected into the flask. After 1 minute, the reaction is quenched by adding $1 \mathrm{~mL}$ of dodecanethiol (DDT) and cooling to room temperature using compressed air on the outside of the flask. The obtained dark solution is then opened to air and precipitated by addition of ethanol and then centrifuged. The supernatant is discarded and the pellet redispersed in toluene. The cleaning procedure is repeated two more times. At the last step, we perform a selective precipitation to split the batch over three fractions. To do so, the solution in pure toluene is first centrifuged without the addition of non-solvent to get rid of the lamellar phase resulting from the complexation of mercury with oleylamine. In this case, only the stable solution is kept while the grey precipitate is discarded. Then, a limited amount of ethanol is added and the solution centrifuged. The pellet will become fraction 2, while the remaining particles in solution are further precipitated by the addition of additional ethanol. We then store fraction 2 in an air free glove box. The nanoparticles in this fraction are $4.5 \mathrm{~nm} \pm 0.5 \mathrm{~nm}$, measured by TEM, see Figure S1a. The intraband absorption peak is at $2698 \mathrm{~cm}^{-1}$, see Figure S1b.

Medium HgSe CQDs: The method for small HgSe CQDs is followed, with 4 minutes between injection of the Se solution and quenching. The pellet from selective precipitation contains particles $5.8 \mathrm{~nm} \pm 0.5 \mathrm{~nm}$ in diameter measured by TEM, see Figure S1c. The intraband absoption peak is at $2245 \mathrm{~cm}^{-1}$, see Figure S1d.

Large HgSe CQDs: For large HgSe CQD, we use $0.1 \mathrm{~g}$ of $\mathrm{Hg}(\mathrm{OAc})_{2}$ with $4 \mathrm{~mL}$ of $\mathrm{OA}$ and $10 \mathrm{~mL}$ of OLA. The solution is degassed at $85^{\circ} \mathrm{C}$ for $30 \mathrm{~min}$. Meanwhile we mix $0.13 \mathrm{~g} \mathrm{SeS}_{2}$ with $2 \mathrm{~mL}$ of oleylamine and sonicate it until it turns brown. The $\mathrm{Hg}(\mathrm{OAc})_{2}$ solution is placed under $\mathrm{Ar}$ and the temperature set at $90^{\circ} \mathrm{C}$. The Se precursor is injected and the reaction is quenched after 6 minutes by adding $1 \mathrm{~mL}$ of DDT and removing the heating mantle. The obtained dark solution is then precipitated by addition of ethanol and then centrifuged. The cleaning procedure is repeated two more times. The obtained nanoparticles have diameters in the 15-17 $\mathrm{nm}$ range according to TEM, see see Figure S1e. The intraband absorption peak is at $986 \mathrm{~cm}^{-1}$, see Figure S1f.

Extra Large HgSe CQDs: For extra-large HgSe CQDs, we use $0.1 \mathrm{~g}$ of $\mathrm{Hg}(\mathrm{OAc})_{2}$ with $4 \mathrm{~mL}$ of $\mathrm{OA}$ and $10 \mathrm{~mL}$ of OLA. The solution is degassed at $85^{\circ} \mathrm{C}$ for $30 \mathrm{~min}$. Meanwhile we mix $0.13 \mathrm{~g} \mathrm{SeS}_{2}$ with $2 \mathrm{~mL}$ of oleylamine and sonicate it until it turns brown. The $\mathrm{Hg}(\mathrm{OAc})_{2}$ solution is placed under $\mathrm{Ar}$ and heated to $120^{\circ} \mathrm{C}$. The Se precursor is injected and after $20 \mathrm{~min}, 1 \mathrm{~mL}$ of DDT is added and the heating mantle is removed. The obtained dark solution is then precipitated by addition of ethanol and then centrifuged. The cleaning procedure is repeated two more times. The obtained nanoparticles have a diameter in the $20 \mathrm{~nm}$ range according to TEM, see Figure S1g. The intraband absorption peaks at $380 \mathrm{~cm}^{-1}$, see Figure $\mathrm{S} 1 \mathrm{~h}$.

Ligand exchange procedure: To prepare thin films of nanoparticles with different capping ligands, we use solid state ligand exchange (ie on-film ligand exchange). We first drop-cast the QD solution on the substrate. When the solvent is completely evaporated, ligand exchange is performed on the sample to remove the DDT and replace it by EDT, $\mathrm{S}^{2-}$ or $\mathrm{As}_{2} \mathrm{~S}_{3}$. This technique consists in dipping the film into the solution containing the new ligands for $1.5 \mathrm{~min}$. The film is then rinsed by dipping it in ethanol for 30 seconds. The EDT, $\mathrm{S}^{2-}$ and $\mathrm{As}_{2} \mathrm{~S}_{3}$ solutions were prepared by mixing respectively EDT, $\mathrm{Na}_{2} \mathrm{~S}$ and a saturated solution of $\mathrm{As}_{2} \mathrm{~S}_{3}$ in butylamine saturated solution with ethanol (1 wt.\%). To get a thicker film, this step was repeated 3 to 4 times.

Material characterization: For TEM imaging, the solution of CQD is drop-cast on a copper TEM grid and dried overnight under vacuum. The TEM images are then obtained using a JEOL 2010 microscope operated at $200 \mathrm{kV}$. Room temperature IR optical absorption is measured using a Bruker Vertex 70 spectrometer. A Globar source equivalent to a $700^{\circ} \mathrm{C}$ blackbody is used as source with a DTGS detector. Spectra are typically acquired between 
$10000 \mathrm{~cm}^{-1}$ and $380 \mathrm{~cm}^{-1}$ with a $4 \mathrm{~cm}^{-1}$ resolution and averaging over 64 spectra. Measurements of the material with DDT ligand are made in an ATR configuration, while we use transmission configuration for ligand exchanged films. In this case we use $\mathrm{CaF}_{2}$ or undoped $\mathrm{Si}$ substrates. For low temperature infrared spectra we used a He gas exchange cryostat with ZnSe windows and conduct the measurements in a transmission configuration. The FTIR is a Bruker IFS66v with optical components similar to the ones in the Vertex 70.

\section{Transport measurements}

Electrode fabrication: Electrodes are fabricated using standard optical lithography methods. Briefly the surface of a $\mathrm{Si} / \mathrm{SiO}_{2}$ (400 nm thick) wafer is cleaned by sonication in acetone. The wafer is rinsed with isopropanol and finally cleaned using a $\mathrm{O}_{2}$ plasma. AZ5214 resist is spincoated and baked at $110^{\circ} \mathrm{C}$ for $90 \mathrm{~s}$. The substrate is exposed under UV through a pattern mask for $2 \mathrm{~s}$. The film is further baked at $125^{\circ} \mathrm{C}$ for $2 \mathrm{~min}$ to invert the resist. Then a $40 \mathrm{~s}$ flood exposure is performed. The resist is developed using a bath of AZ726 for $32 \mathrm{~s}$, before being rinsed with pure water. We then deposit a $3 \mathrm{~nm} \mathrm{Cr}$ layer and a $40 \mathrm{~nm}$ gold layer using a thermal evaporator. The lift-off is performed by dipping the film for $1 \mathrm{~h}$ in acetone. The electrodes are finally rinsed using isopropanol and dried by air flow. The electrodes are $2 \mathrm{~mm}$ long and spaced by $20 \mu \mathrm{m}$.

Electrolyte preparation: $50 \mathrm{mg}$ of $\mathrm{LiClO}_{4}$ are mixed with $230 \mathrm{mg}$ of PEG on a hot plate in an Ar filled glove box at $170^{\circ} \mathrm{C}$ for $2 \mathrm{~h}$.

Transistor fabrication: We start from a solution of HgSe CQDs capped with DDT ligand. We prepare a solution of $\mathrm{S}^{2-}$ anions from a $\mathrm{Na}_{2} \mathrm{~S}$ dissolved in $\mathrm{N}$-methyl formamide. The CQDs dispersed in toluene are mixed with the $\mathrm{S}^{2-}$ solution until a phase transfer is observed. The colorless toluene phase is discarded and the polar bottom phase is further cleaned by adding hexane. After three hexane cleanings, the solution is precipitated by addition of ethanol and centrifuged to form a pellet. The latter is redispersed in fresh $\mathrm{N}$-methyl formamide. This solution is dropcast onto the electrodes on a hot plate at $100^{\circ} \mathrm{C}$. Meanwhile the electrolyte is softened at $100^{\circ} \mathrm{C}$. The melted electrolyte is now clear and is brushed on the CQD film. A copper grid is then deposited on the top of the electrolyte and used as top gate.

Transistor measurements: Electrical measurements are conducted in air using two Keithley 2400 as source-meters. Transistor measurements are conducted by biasing the drain source under low bias (10 or $50 \mathrm{mV})$ and applying a varying gate bias. The gate step bias is $1 \mathrm{mV}$ at $2 \mathrm{mV} . \mathrm{s}^{-1}$ for electrolyte gating. Temperature dependent electrical measurements were carried out inside a He-flow cryostat of $1.5 \mathrm{~K}$ base temperature, using a low current sourcemeter K2634B for low signal measurements, with +/- $0.1 \mathrm{pA}$.

\section{RESULTS AND DISCUSSION}

We synthesized and studied the electronic properties of four sizes of HgSe CQDs: $4.5 \mathrm{~nm}$ (small), $5.9 \mathrm{~nm}$ (medium), $17 \mathrm{~nm}$ (large), and $20 \mathrm{~nm}$ (extra-large), with decreasing confinement. Figure 1 shows the absorption (1-transmission) spectra for each of the sizes capped with dodecanethiol and a transmission electron microscope (TEM) image of the $5.9 \mathrm{~nm}$ diameter particles. The absorption spectra peaks span in the mid- and far-IR range at 3.7, 4.4, 10.4, and $26 \mu \mathrm{m}(11 \mathrm{THz})$, for the 4 sizes, respectively. The latter is currently the reddest absorbance reported so far for colloidal materials. For application in photodetection, a ligand exchange step is necessary to replace the initial long insulating ligands (dodecanethiol). Here, we focus on three different surface chemistries: 1,2-ethanedithiol (EDT), sulfide ions ${ }^{27}$ and $\mathrm{As}_{2} \mathrm{~S}_{3},{ }^{28,29}$ following previous reports of photoconductivity using these ligands. Additional absorption data for each size after the different ligand exchanges, as well as TEM images are provided in Figure S1. 


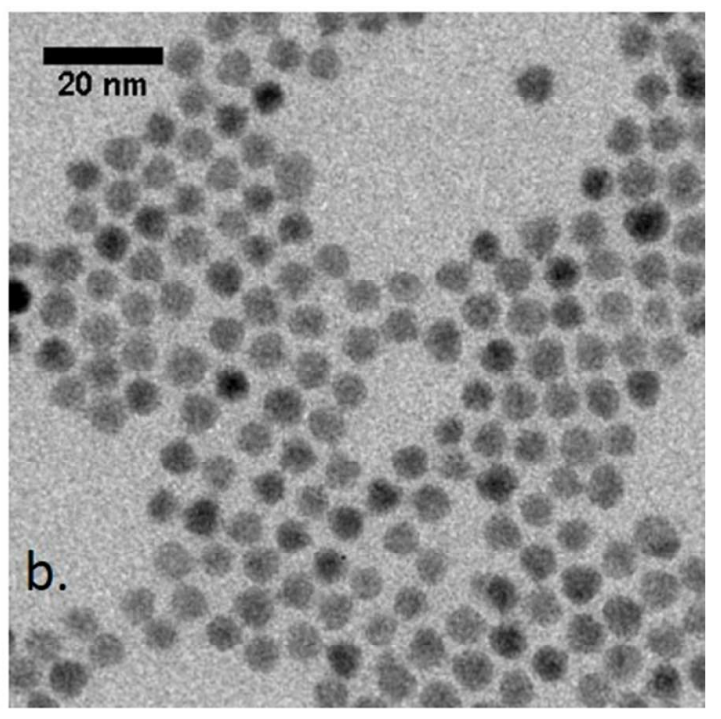

Figure 1 a. Absorption spectra for four sizes of HgSe CQD. b. TEM image of medium size HgSe CQD.

X-ray photoemission measurements are conducted on freshly prepared HgSe CQD films at the Tempo beamline of synchrotron Soleil to investigate the electronic spectrum of HgSe CQDs as a function of their sizes and surface chemistries. Details on the analysis of the photoemission measurements are given in the SI. A typical overview of the photoemission spectrum for HgSe CQDs is given in Figure 2a. We clearly observe four core level contributions from $\mathrm{Hg}$, Se, S and C (from the ligands). We then focus on the $\mathrm{Hg} 4 \mathrm{f}$ core level photoemission observed as a doublet with $4.06 \mathrm{eV}$ spin orbit splitting, as shown in Figure $2 \mathrm{~b}$. After subtraction of the background, we fit the peaks with two Voigt curves, constraining the doublet pairs to have the same full width at half maximum (FWHM), and a spin-orbit splitting of $4.06 \mathrm{eV}$. The $4 \mathrm{f}_{7 / 2}$ peak is found with contributions at $100.3 \mathrm{eV}$ and $101 \mathrm{eV}$. To clarify the origin of the two contributions, we first change the incident photon energy to tune the photoelectron escape depth. As the kinetic energy of the photoelectron increases, the escape depth increases ${ }^{30}$ and the relative amplitude of the second peak is reduced, as shown in Figure S4b. In addition, the relative weight of the second peak is also reduced with increasing CQD size, in relation with a decrease in the surface to volume ratio. We therefore attribute this second peak to a surface contribution. This is supported by EDX measurements (see Figure S2), which systematically show the CQDs to be Hg-rich, consistent with previous observations. ${ }^{31}$ The excess $\mathrm{Hg}$ atoms are located on the surface and are bonded to $S$ atoms from the ligands, whereas the bulk $\mathrm{Hg}$ atoms are bonded to Se atoms. Because of the higher electronegativity of S compared to $\mathrm{Se}$, the $\mathrm{Hg}$ atoms at the surface are less electron rich, which results in the observed shift to higher binding energies (BE) for the surface peak.

Along with this surface contribution of the $\mathrm{Hg}$ core level, we notice a shift of the bands and vacuum level while tuning the capping ligands. The latter can be as large as $\approx 0.5 \mathrm{eV}$, as shown in Figure 2c. This shift of the vacuum level with different ligands is a direct evidence that the change of doping with surface chemistry is the result of the band bending associated with the surface dipoles. ${ }^{16,18,20}$ High resolution photoemission also unveils features that cannot be observed through an electrochemical approach. The ratio of the peak areas from the fit of the $\mathrm{Hg} 4 \mathrm{f}$ state gives the fraction of the volume affected by the surface dipoles. We estimate the length over which the band bending is occurring to be $0.9 \mathrm{~nm}$ for the small CQD. This is consistent with the Debye length, $\lambda=\sqrt{\frac{\varepsilon_{0} \varepsilon_{r} k_{b} T}{n_{\text {film }} e^{2}}} \approx 0.9 \mathrm{~nm}$ where $k_{b} T$ is the thermal energy (25 meV at room temperature) and $n_{\text {film }}$ the film carrier density $\left(2.6 \times 10^{19} \mathrm{~cm}^{-3}\right.$ for small CQD), over which the screening of the dipole electric field occurs. 

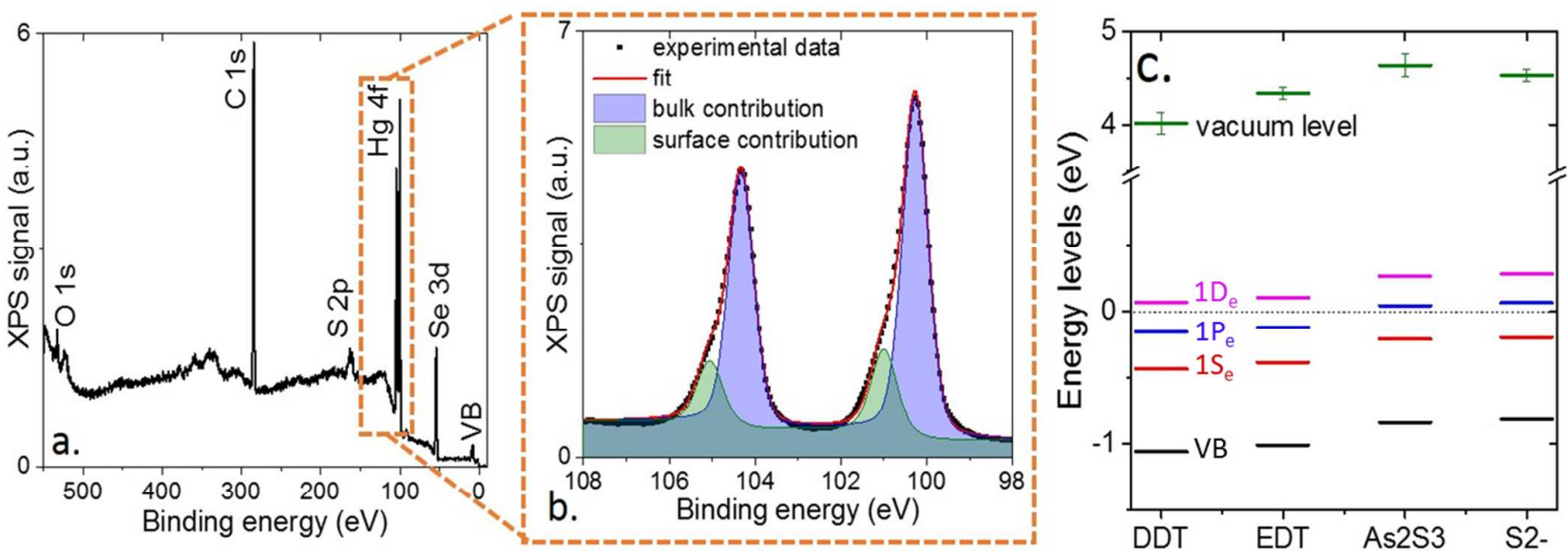

Figure 2: a. XPS signal overview for a HgSe CQD film. The incident photon energy is $600 \mathrm{eV}$. b. $\mathrm{Hg} 4 \mathrm{f}$ core level photoemission spectrum for the medium HgSe CQDs capped with $\mathrm{As}_{2} \mathrm{~S}_{3} \mathrm{C}$. Electronic spectrum (black is the valence band, red is the $1 S_{e}$ state, blue is the $1 P_{e}$ state, pink is the $1 D_{e}$ state, and green is the vacuum level) for medium HgSe CQDs capped with four different ligands. Zero on the energy scale corresponds to the Fermi level. The error bars have been obtained by determining the work function using different methods.

We then reconstruct, on an absolute energy scale, the electronic spectrum of the HgSe CQDs for different capping ligands and nanocrystal sizes. Additional details of the analysis are provided in the SI. The work function (Fermi level vs vacuum) is extracted from the cut-off of the secondary electron emission. The valence band energy with respect to the Fermi energy is determined from the low BE cutoff of the XPS spectrum. To determine the states above the Fermi level, the quasiparticle band gap (Eg) is determined ${ }^{21}$ from the excitation gap $\mathrm{Eg}_{0}$ (optical gap) and is corrected by the exciton binding energy through the relation $E g=E g_{0}+\Sigma$, here we use $\Sigma={\frac{1.8 e^{2}}{4 \pi \varepsilon_{0} \varepsilon_{r} r}}^{18,22}$ with $\varepsilon_{0}$ the vacuum permittivity, $\varepsilon_{r}$ the HgSe relative permittivity, e the proton charge and $r$ the particle radius. The Coulomb polarization introduces a small correction of 12 to $50 \mathrm{meV}$ to the optical gap depending on the CQD size. This gives the energy of the $1 S_{e}$ state with respect to the valence band. The intraband gap is determined using IR spectroscopy and allows to locate the $1 \mathrm{P}_{\mathrm{e}}$ state with respect to the $1 \mathrm{~S}_{\mathrm{e}}$ state. Unlike the $\mathrm{HgS}$ case, no optical signature of the $1 \mathrm{D}_{\mathrm{e}}$ state is observed for HgSe here, ${ }^{12}$ and we extrapolate the energy of the $1 \mathrm{D}_{\mathrm{e}}$ level with respect to the $1 \mathrm{P}_{\mathrm{e}}$ level using a three band $k \cdot p$ model previously described. ${ }^{22,31,32}$ This simple model considers a nondispersive heavy hole band and symmetric (i.e. same mass with opposite sign) $\Gamma_{6}$ and $\Gamma_{8}$ bands. The associated Hamiltonian includes two parameters: the $\Gamma_{6}-\Gamma_{8}$ splitting (the negative band gap of bulk $\mathrm{HgSe}$, $\left.E_{G}^{\text {bulk }}{ }^{14,15}\right)$ and the Kane energy $\left(E_{p}\right)$. To accurately determine these two parameters, we fit the $\mathrm{k} \cdot \mathrm{p}$ dispersion to all the experimental points available from the literature ${ }^{1,13,27}$ for the inter- and intra-band gaps as a function of size, giving $E_{g}^{\text {bulk }}=-0.2 \mathrm{eV}$, and $E_{p}=10.27 \mathrm{eV}$ at room temperature. The similarity with previously reported values for these two parameters is good for such a simplistic model. ${ }^{28,29}$ The reconstructed spectra for the small, medium, and large HgSe CQDs are given in Figure 2c and S3.

Using the reconstructed electronic spectra and the state degeneracies, we can estimate the number of electrons per particle $n_{C Q D}{ }^{1}$ The small size (figure S3a) corresponds to the case where the $1 S_{\mathrm{e}}$ state is being filled. The electron filling per CQD is in the $n_{C Q D} \approx 0.2-2$ electron(s) range ${ }^{16}$. In the medium CQDs, the Fermi level is near or above the $1 \mathrm{P}_{\mathrm{e}}$ state and $\mathrm{n}_{\mathrm{CQD}}$ is between 2 and 8 . For the large CQDs (Figure S3c), the Fermi level is above the $1 D_{e}$ state meaning that the filling reaches $n_{C Q D} \approx 18$ electrons per CQD. As a result the individual nanoparticles acquire a more and more metallic character as their size increases. On the other hand, the carrier density of the film $\left(=n_{C Q D}\right.$, divided by the particle volume, multiplied by the packing volume fraction) actually decreases slightly with increasing CQD size. We estimate the film carrier 
density to be $n_{\text {film }} \approx 2.6 \times 10^{19} \mathrm{~cm}^{-3}$ for small CQDs and $n_{\text {film }} \approx 4.5 \times 10^{18} \mathrm{~cm}^{-3}$ for the largest CQDs, while the volume ratio between these two sizes of particles is about 50 . This observation is consistent with the redshift of the mid IR peak with increasing size shown in Figure 1a, as the plasmon peak energy $w_{p}$ scales with $\sqrt{n_{\text {film }}}$. As the CQD size is increased, we thus expect to observe, at the nanoparticle level, a transition from semiconductor behavior, where single electrons drive the optical and transport properties, toward a more metallic behavior, where collective effects should emerge. Moreover, this transition strongly differs from the conventional metal-insulator transitions observed in bulk or other strongly doped semiconductor nanoparticles, ${ }^{33}$ since it occurs at a fairly constant film doping level $\left(\mathrm{n}_{\text {film }}\right)$.

In the final part of the paper, we investigate the signature of the transition to metallic behavior in the optical and transport properties of the film. Figure 3a and S5 show that as the system is cooled, we observe a small redshift of the interband absorption, as reported previously for $\mathrm{HgTe}^{32}$, and a blueshift of the intraband/plasmonic peak. ${ }^{13}$ This blue shift of the intraband peak with decreasing temperature becomes weaker as the CQD size is increased, as shown in Figure $3 \mathrm{~b}$. To rationalize this observation, we consider that the mid IR peak results from the intraband transition only if the $1 \mathrm{P}_{\mathrm{e}}$ state remains unfilled (ie only for the small CQDs). For the larger CQDs, the intraband absorption is weakened and the mid IR peak becomes plasmonic in nature, as recently observed in $\mathrm{HgS}^{22}$ in n-doped Si nanoparticles, ${ }^{34,35}$ or in strongly doped quantum wells. ${ }^{23}$ When the absorption is dominated by the intraband transition, its shift with temperature is similar to that observed for intersubband transitions in doped quantum wells. ${ }^{36}$ In this case, the main temperature dependence results from the depolarization shift, which scales like the difference in populations of the ground and excited states. ${ }^{36}$ That excitation is thermally activated and leads to the strong temperature dependence observed for the small CQDs. As the CQD size is increased, the transition loses its intraband character and the absorption is dominated by the collective plasmon excitation. ${ }^{37}$ The plasma frequency $w_{p}$ depends on the square root of the carrier density, which has a weak temperature dependence. This explains the saturation of the shift measured for the large HgSe CQD. 

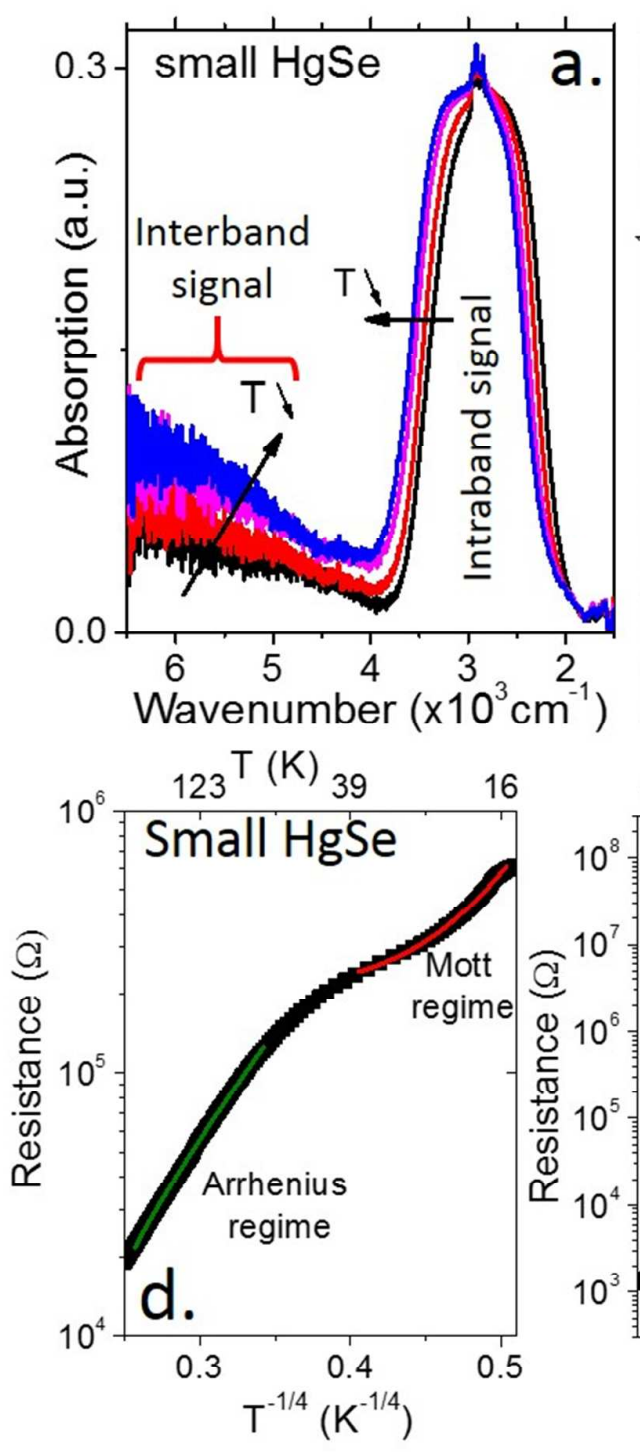
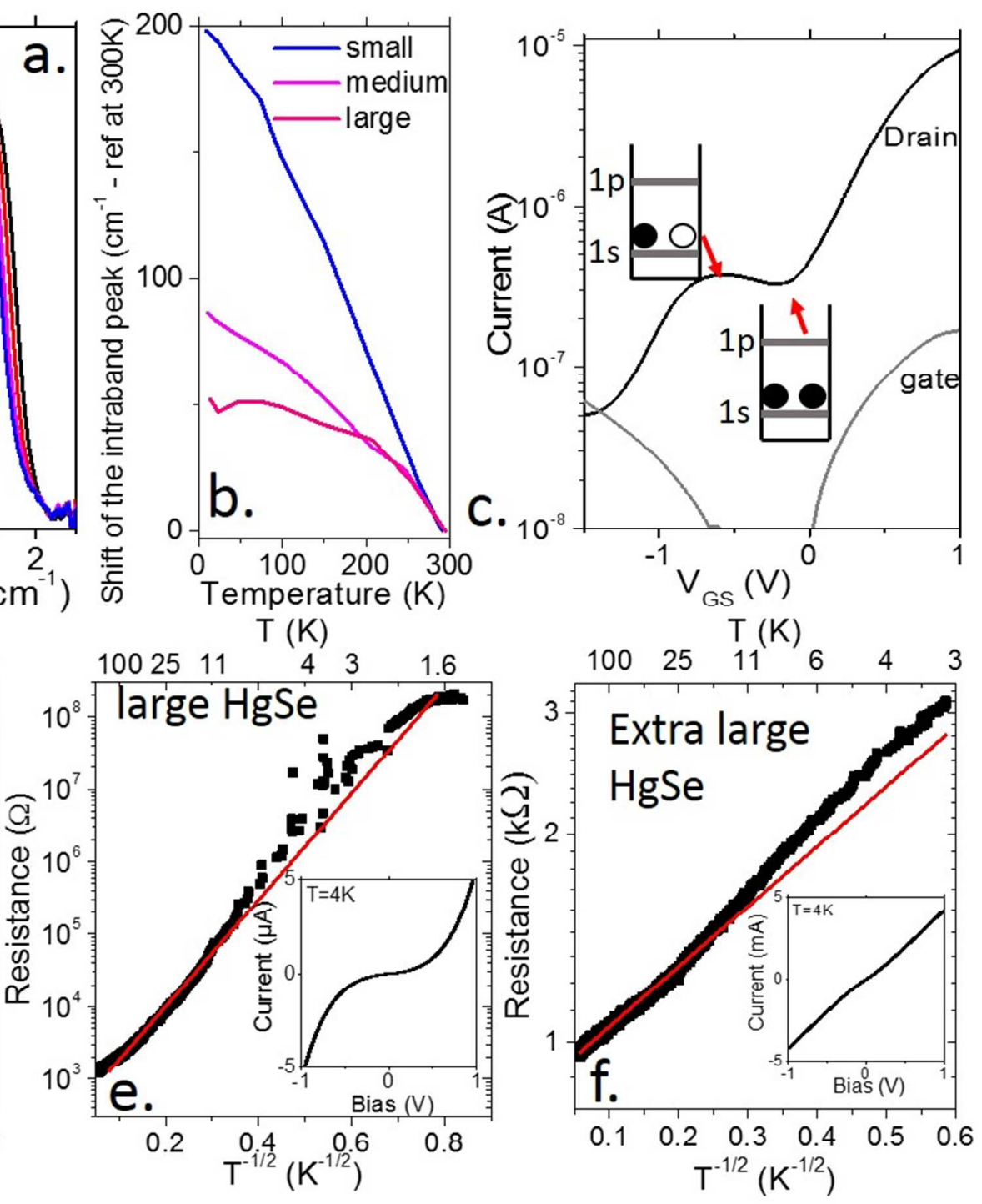

Figure 3 a. Absorption spectra for the small size CQDs at temperatures from 300 down to $4 \mathrm{~K}$. b. shift of the intraband/plasmonic peak as a function of temperature for three sizes of HgSe CQDs. c. transfer curve (drain and gate current vs gate bias) for a film of medium HgSe CQDs capped with $\mathrm{S}^{2-}$. d. Resistance of a film of small HgSe CQDs capped with $S^{2-}$ as a function of temperature. e. Resistance of a film of large HgSe CQDs capped with $S^{2-}$ as a function of temperature. The inset is an IV curve measured at $4 \mathrm{~K}$ for a film of large HgSe CQDs capped with $S^{2-}$. $f$. Resistance of a film of extra large $\mathrm{HgSe} C Q D$ s capped with $\mathrm{S}^{2-}$ as a function of temperature. The inset is an IV curve measured at $4 \mathrm{~K}$ from a film of extra-large HgSe CQD film capped with $S^{2-}$ ligands. The red lines in $d-f$ are power law fits (linear on the respective $x$-axes), as described in the text.

Confinement also strongly affects the conductance of the film. We used ion-gel gated transistors to characterize electronic transport in films of the HgSe CQDs, ${ }^{38,39}$ see figure S6 for a scheme of the device. This gating method combines several advantages. The large capacitance $\left(\approx \mu \mathrm{F} . \mathrm{cm}^{-2}\right)$ associated with the electrolyte allows injection of several carriers per CQD. The devices are also air operable and can be used to gate thick films, thanks to percolation of the ions through the film ${ }^{40}$. For small and medium CQDs, we measure a strong modulation of the current with gate bias, shown in Figure 3c. The transfer curve shows an increase in conductance as electrons are injected (positive gate bias), which confirms the n-type nature of the material. The non-monotonic behavior of this increase results from Pauli blockade, ${ }^{1}$ which leads to a local minimum as the $1 S_{e}$ state is completely filled. For the large and extra-large CQDs, we observe increased conductance but a loss of the gate dependence, as shown in Figure S7-S9. We attribute the loss of the gate effect to the reduced surface to volume ratio, which reduces the efficiency of ion gating. 
Although the overall carrier density in the films does not increase with particle size, the conductivity does. This points to higher carrier electron mobility, which arises partly from fewer hops between contacts, but predominantly from better overlap between the higher level 1Pe and 1De states. We have reported electron mobilities up $90 \mathrm{~cm}^{2} \mathrm{v}^{-1} \mathrm{~s}^{-1},{ }^{1}$ while much lower values are typical for CQD films. ${ }^{22}$

The temperature dependence of the current also reveals the higher density of states for HgSe CQDs with increasing size. At high temperature $(T>100 \mathrm{~K})$, the current follows an Arrhenius dependence with a low activation energy ( $25 \mathrm{meV}$ for the small CQDs and $5 \mathrm{meV}$ for the large ones), see Figure 3d. At lower temperature, the transport switches from nearest neighbor to variable range hopping (VRH). In variable range hopping, the logarithm of the conductivity follows a power law with temperature with an exponent of either -0.25 (Mott; $\sigma \propto \exp \left(-\left(T_{M} / T\right)^{1 / 4}\right)$ ) or -0.5 (Efros-Shklovskii $\left.-\sigma \propto \exp \left(-\left(T_{E S} / T\right)^{1 / 2}\right)\right)$. $\mathrm{T}_{\mathrm{M}}$ and $\mathrm{T}_{\mathrm{ES}}$ are constants that depend on the CQD, ligand, and film morphology, obtained here as fitting parameters. For the small dots, -0.25 gives the best fit, as shown in Figure $3 \mathrm{~d}$ and Figure 57 , while for the larger dots, 0.5 gives the best agreement, as shown in Figure 3e-f, S8 and S9.

That the conductivity exhibits variable range hopping temperature dependence shows that while the individual particles appear metallic, the overall film remains on the semiconductor/insulator side of the insulator-metal transition. This is due to limited interparticle coupling, despite the use of short $S^{2-}$ capping ligands. The change of the power law, from Mott to Efros-Shklovskii (ES) with increasing CQDs size, suggests that there is a transition from a transport mechanism. The energy scale is defined, in the Mott case (corresponding to small CQDs, see Figure 4a) by either disorder due to the size distribution or, in the case of completely filled levels, the interlevel spacing and switches, in the ES case (large CQDs, Figure $4 b)$, to an energy scale defined by the Coulomb energy. Unlike Mott $\mathrm{VRH}$, that can be only observed in semi-conducting systems, ES VRH can also be observed in metallic systems such as gold metallic nanoparticle films coupled through short capping ligands. ${ }^{41,42}$ Thus, the transition from Mott to ES VRH is consistent with a transition of the individual CQDs from the semiconducting (small CQDs) to metallic particles (large CQDs), even though the films remain globally insulating because of the weak inter CQD coupling.

Cross-overs from Mott to ES VRH have been observed in other CQD systems. In CdSe CQDs, such a crossover was observed when CQD films were charged via electrolyte. ${ }^{43}$ Similarly, Chen et al. observed a metal insulator transition, with p-doped Si nanoparticles. ${ }^{33}$ In these two cases, the tuned parameter is the carrier density. In contrast, in this work with HgSe CQDs, it is the confinement that is tuned. This is equivalent changing the Bohr radius. Another striking difference in this work, compared to previous reports on granular wide band gap semiconductors, ${ }^{44}$ comes from the estimation of the coherence length $\xi=\frac{2.8 e^{2}}{\varepsilon_{0} \varepsilon_{r} k_{b} T_{E S}}=136 \mathrm{~nm}$, assuming $\varepsilon_{\mathrm{r}}=16$ for large HgSe CQDs. ${ }^{43}$ This value for $\xi$ is one decade larger than typical values for Si CQDs, ${ }^{33}$ and a factor 100 larger than that for CdSe CQDs. ${ }^{43}$ We expect the scaling of the coherence length to follow ${ }^{43} R_{C Q D} / \xi \propto \Delta E / k_{b} T$ with $\Delta E$ the interlevel spacing, such that the longer coherence length measured for HgSe CQDs here reflects the larger density of states (smaller $\triangle \mathrm{E}$ ) compared to CdSe and $\mathrm{Si}$. This very long coherence length demonstrates that narrow band gap CQDs are a promising platform for the emergence of coherent transport at the mesoscale. 

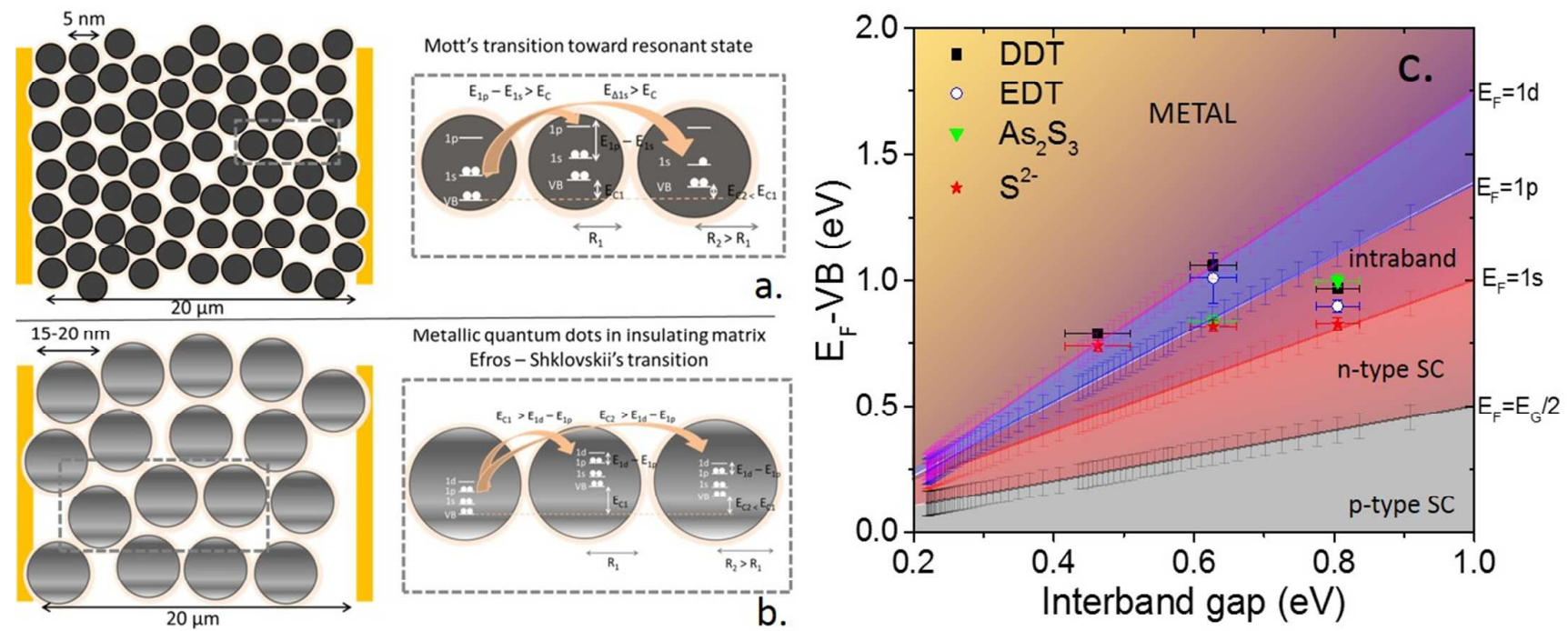

Figure 4 a. Scheme of a film of small HgSe CQDs and of the transition between two resonant states according to Mott VRH. b. Scheme of a film of large HgSe CQDs and of the transition between two CQDs. In ES VRH, electron transfer must overcome the Coulomb energy. c. Fermi energy with respect to the valence band as a function of the interband gap. The solid lines correspond to the mid interband gap, $1 S_{e}$, $1 P_{e}$ and $1 D_{e}$ states from the 3 band $k \cdot p$ simulation.

Finally, we summarize the results for the different sizes and ligands into a phase diagram, ${ }^{19}$ depicted in Figure 4c, which gives the position of the Fermi energy with respect to the valence band as a function of the interband gap (confinement energy). This type of diagram is of utmost interest in designing devices based on this material and especially for the choice of the metal electrodes. The degenerately doped nature of the HgSe CQD is highlighted by the lack of experimental points within the two lower zones of the diagram, corresponding to cases where the Fermi level remains within the interband gap. As the CQD size increases, we observe a relaxation of confinement and the doped semiconductor behavior becomes more metallic at the single particle level, resulting from the filling of higher energy states.

\section{CONCLUSION}

In summary, we have synthesized a series of HgSe CQDs with decreasing confinement. Using a combination of IR and photoemission spectroscopy, we have been able to determine the electronic spectrum on an absolute energy scale for three sizes and four different ligands, representative of those reported in photoconductive devices. We confirmed that the ligands affect the electronic spectrum of the HgSe CQDs through band bending resulting from the surface dipole. We determine a length on the order of $1 \mathrm{~nm}$, over which the surface dipole affects the local potential within the CQDs. We propose a phase diagram which can easily be used to determine the population within HgSe CQDs. As the CQD size raises from $4.5 \mathrm{~nm}$ to $20 \mathrm{~nm}$ the population increases from 2 to more than 18 electrons per particle. This increase of the CQD carrier density leads to a change of behavior from semiconducting (intraband transition + Mott $\mathrm{VRH}$ ) to metallic behavior where collective effects (plasmonic absorption and ES VRH) predominate. The film itself remains semiconductive, as the inter-CQD coupling remains too weak to observe band-like transport.

\section{SUPPORTING INFORMATIONS}

The Supporting Information is available free of charge on the ACS Publications website. 
It includes details on the nanocrystal synthesis, the photoemission procedure and complementary transport data.

\section{ACKNOWLEDEGMENTS}

We acknowledge the use of clean-room facilities from the "Centrale de Proximité Paris-Centre". EL thanks the support ERC starting grant blackQD. We thank Agence Nationale de la Recherche for funding through grant Nanodose and H2DH. This work has been supported by the Region lle-de-France in the framework of DIM Nano-K. This work was supported by French state funds managed by the ANR within the Investissements d'Avenir programme under reference ANR-11-IDEX-0004-02, and more specifically within the framework of the Cluster of Excellence MATISSE.

\section{REFERENCES}

(1) Lhuillier, E.; Scarafagio, M.; Hease, P.; Nadal, B.; Aubin, H.; Xu, X. Z.; Lequeux, N.; Patriarche, G.; Ithurria, S.; Dubertret, B. Infrared Photodetection Based on Colloidal Quantum-Dot Films with High Mobility and Optical Absorption up to THz. Nano Lett. 2016, 16, 1282-1286.

(2) Talapin, Dmitri V.; Lee, Jong Soo; Kovalenko, Maksym V.; Shevchenko, Elena V. Prospects of Colloidal Nanocrystals for Electronic and Optoelectronic Applications. Chem Rev 2010, 110 (1), 389458.

(3) Konstantatos, G.; Sargent, E. H. Colloidal Quantum Dot Optoelectronics and Photovoltaics; Cambridge University Press, 2013.

(4) Tang, Xin; Wu, Guang fu; Chiu Lai, King Wai. Plasmon Resonance Enhanced Colloidal HgSe Quantum Dot Filterless Narrowband Photodetectors for Mid-Wave Infrared. J Mater Chem C 2017, 5, 362-369.

(5) Keuleyan, S.; Lhuillier, E.; Brajuskovic, V.; Guyot-Sionnest, P. Mid-Infrared HgTe Colloidal Quantum Dot Photodetectors. Nat. Photonics 2011, 5, 489-493.

(6) Lhuillier, E.; Guyot-Sionnest, P. Recent Progresses in Mid Infrared Nanocrystal Based Optoelectronics. IEEE J. Sel. Top. Quantum Electron. 2017, PP, 1-1.

(7) Kershaw, S. V.; Susha, A. S.; Rogach, A. L. Narrow Bandgap Colloidal Metal Chalcogenide Quantum Dots: Synthetic Methods, Heterostructures, Assemblies, Electronic and Infrared Optical Properties. Chem. Soc. Rev. 2013, 42, 3033-3087.

(8) Huo, N.; Gupta, S.; Konstantatos, G. MoS2-HgTe Quantum Dot Hybrid Photodetectors beyond 2 Mm. Adv. Mater. 2017, 29, n/a-n/a.

(9) Chen, M.; Lu, H.; Abdelazim, N. M.; Zhu, Y.; Wang, Z.; Ren, W.; Kershaw, S. V.; Rogach, A. L.; Zhao, N. Mercury Telluride Quantum Dot Based Phototransistor Enabling High-Sensitivity RoomTemperature Photodetection at $2000 \mathrm{Nm}$. ACS Nano 2017, 11, 5614-5622.

(10) Chen, M.; Shao, L.; Kershaw, S. V.; Yu, H.; Wang, J.; Rogach, A. L.; Zhao, N. Photocurrent Enhancement of HgTe Quantum Dot Photodiodes by Plasmonic Gold Nanorod Structures. ACS Nano 2014, 8, 8208-8216.

(11) Jeong, K. S.; Deng, Z.; Keuleyan, S.; Liu, H.; Guyot-Sionnest, P. Air-Stable N-Doped Colloidal HgS Quantum Dots. J. Phys. Chem. Lett. 2014, 5, 1139-1143.

(12) Yoon, B.; Jeong, J.; Jeong, K. S. Higher Quantum State Transitions in Colloidal Quantum Dot with Heavy Electron Doping. J. Phys. Chem. C 2016, 120, 22062-22068.

(13) Deng, Zhiyou; Jeong, Kwang Seob; Guyot-Sionnest, Philippe. Colloidal Quantum Dots Intraband Photodetectors. ACS Nano 2014, 8 (11), 11707-11714.

(14) von Truchseß, M.; Pfeuffer-Jeschke, A.; Becker, C. R.; Landwehr, G.; Batke, E. Electronic Band Structure of HgSe from Fourier Transform Spectroscopy. Phys. Rev. B 2000, 61, 1666-1669.

(15) Svane, A.; Christensen, N. E.; Cardona, M.; Chantis, A. N.; van Schilfgaarde, M.; Kotani, T. Quasiparticle Band Structures of \$lensuremath\{lbeta\}\$-HgS, HgSe, and HgTe. Phys. Rev. B 2011, 84, 205205.

(16) Robin, A.; Livache, C.; Ithurria, S.; Lacaze, E.; Dubertret, B.; Lhuillier, E. Surface Control of Doping in Self-Doped Nanocrystals. ACS Appl. Mater. Interfaces 2016, 8, 27122-27128.

(17) Shen, G.; Guyot-Sionnest, P. HgS and HgS/CdS Colloidal Quantum Dots with Infrared Intraband Transitions and Emergence of a Surface Plasmon. J. Phys. Chem. C 2016, 120, 11744-11753.

(18) Brown, P. R.; Kim, D.; Lunt, R. R.; Zhao, N.; Bawendi, M. G.; Grossman, J. C.; Bulović, V. Energy Level Modification in Lead Sulfide Quantum Dot Thin Films through Ligand Exchange. ACS Nano 2014, 8, 5863-5872. 
(19) Miller, E. M.; Kroupa, D. M.; Zhang, J.; Schulz, P.; Marshall, A. R.; Kahn, A.; Lany, S.; Luther, J. M.; Beard, M. C.; Perkins, C. L.; van de Lagemaat, J. Revisiting the Valence and Conduction Band Size Dependence of PbS Quantum Dot Thin Films. ACS Nano 2016, 10, 3302-3311.

(20) Kroupa, D. M.; Vörös, M.; Brawand, N. P.; McNichols, B. W.; Miller, E. M.; Gu, J.; Nozik, A. J. (ORCID:0000000171767645); Sellinger, A.; Galli, G.; Beard, M. C. Tuning Colloidal Quantum Dot Band Edge Positions through Solution-Phase Surface Chemistry Modification. Nat. Commun. 2017, 8.

(21) Wang, H.; Lhuillier, E.; Yu, Q.; Zimmers, A.; Dubertret, B.; Ulysse, C.; Aubin, H. Transport in a Single Self-Doped Nanocrystal. ACS Nano 2017, 11, 1222-1229.

(22) Chen, M.; Guyot-Sionnest, P. Reversible Electrochemistry of Mercury Chalcogenide Colloidal Quantum Dot Films. ACS Nano 2017.

(23) Askenazi, B.; Vasanelli, A.; Delteil, A.; Todorov, Y.; Andreani, L. C.; Beaudoin, G.; Sagnes, I.; Sirtori, C. Ultra-Strong Light-matter Coupling for Designer Reststrahlen Band. New J. Phys. 2014, 16, 43029.

(24) Mott, N. F. Metal-Insulator Transition. Rev. Mod. Phys. 1968, 40, 677-683.

(25) Fu, H.; Reich, K. V.; Shklovskii, B. I. Hopping Conductivity and Insulator-Metal Transition in Films of Touching Semiconductor Nanocrystals. Phys. Rev. B 2016, 93, 125430.

(26) Carras, M.; Berger, V.; Marcadet, X.; Vinter, B. Experimental Evidence of a Mott Transition in Highly Doped Two-Dimensional Confined Structures. Phys. Rev. B 2004, 70, 233310.

(27) Nag, A.; Kovalenko, M. V.; Lee, J.-S.; Liu, W.; Spokoyny, B.; Talapin, D. V. Metal-Free Inorganic Ligands for Colloidal Nanocrystals: S2-, HS-, Se2-, HSe-, Te2-, HTe-, TeS32-, OH-, and NH2as Surface Ligands. J. Am. Chem. Soc. 2011, 133, 10612-10620.

(28) Lhuillier, E.; Keuleyan, S.; Zolotavin, P.; Guyot-Sionnest, P. Mid-Infrared HgTe/As2S3 Field Effect Transistors and Photodetectors. Adv. Mater. 2013, 25, 137-141.

(29) Yakunin, S.; Dirin, D. N.; Protesescu, L.; Sytnyk, M.; Tollabimazraehno, S.; Humer, M.; Hackl, F.; Fromherz, T.; Bodnarchuk, M. I.; Kovalenko, M. V.; Heiss, W. High Infrared Photoconductivity in Films of Arsenic-Sulfide-Encapsulated Lead-Sulfide Nanocrystals. ACS Nano 2014, 8, 12883-12894.

(30) Wagner, C. D.; Riggs, W. M.; Davis, L. E.; Moulder, J. F.; Muilenberg, G. E. E. Handbook of X-Ray Photoelectron Spectroscopy; Perkin Elmer Edition, Eden Prairie.; 1979.

(31) Jeong, J.; Yoon, B.; Kwon, Y.-W.; Choi, D.; Jeong, K. S. Singly and Doubly Occupied Higher Quantum States in Nanocrystals. Nano Lett. 2017, 17, 1187-1193.

(32) Lhuillier, E.; Keuleyan, S.; Guyot-Sionnest, P. Optical Properties of HgTe Colloidal Quantum Dots. Nanotechnology 2012, 23, 175705.

(33) Chen, T.; Reich, K. V.; Kramer, N. J.; Fu, H.; Kortshagen, U. R.; Shklovskii, B. I. Metal-Insulator Transition in Films of Doped Semiconductor Nanocrystals. Nat. Mater. 2016, 15, 299-303.

(34) Pi, X.; Delerue, C. Tight-Binding Calculations of the Optical Response of Optimally P-Doped Si Nanocrystals: A Model for Localized Surface Plasmon Resonance. Phys. Rev. Lett. 2013, 111, 177402.

(35) Zhou, S.; Pi, X.; Ni, Z.; Ding, Y.; Jiang, Y.; Jin, C.; Delerue, C.; Yang, D.; Nozaki, T. Comparative Study on the Localized Surface Plasmon Resonance of Boron- and Phosphorus-Doped Silicon Nanocrystals. ACS Nano 2015, 9, 378-386.

(36) Sasagawa, R.; Sugawara, H.; Ohno, Y.; Nakajima, H.; Tsujino, S. Enhancement of Intersubband Transition Energies in GaAs Quantum Wells by Si Delta Doping of High Concentration. Appl. Phys. Lett. 1998, 72, 719-721.

(37) Link, S.; El-Sayed, M. A. Size and Temperature Dependence of the Plasmon Absorption of Colloidal Gold Nanoparticles. J. Phys. Chem. B 1999, 103, 4212-4217.

(38) Lhuillier, E.; Ithurria, S.; Descamps-Mandine, A.; Douillard, T.; Castaing, R.; Xu, X. Z.; Taberna, P.L.; Simon, P.; Aubin, H.; Dubertret, B. Investigating the N- and P-Type Electrolytic Charging of Colloidal Nanoplatelets. J. Phys. Chem. C 2015, 119, 21795-21799.

(39) Lhuillier, E.; Dayen, J.-F.; Thomas, D. O.; Robin, A.; Doudin, B.; Dubertret, B. Nanoplatelets Bridging a Nanotrench: A New Architecture for Photodetectors with Increased Sensitivity. Nano Lett. 2015, 15, 1736-1742.

(40) Puntambekar, A.; Wang, Q.; Miller, L.; Smieszek, N.; Chakrapani, V. Electrochemical Charging of CdSe Quantum Dots: Effects of Adsorption versus Intercalation. ACS Nano 2016, 10, 10988-10999.

(41) Tran, T. B.; Beloborodov, I. S.; Lin, X. M.; Bigioni, T. P.; Vinokur, V. M.; Jaeger, H. M. Multiple Cotunneling in Large Quantum Dot Arrays. Phys. Rev. Lett. 2005, 95, 76806.

(42) Moreira, H.; Yu, Q.; Nadal, B.; Bresson, B.; Rosticher, M.; Lequeux, N.; Zimmers, A.; Aubin, H. Electron Cotunneling Transport in Gold Nanocrystal Arrays. Phys. Rev. Lett. 2011, 107, 176803.

(43) Liu, H.; Pourret, A.; Guyot-Sionnest, P. Mott and Efros-Shklovskii Variable Range Hopping in CdSe Quantum Dots Films. ACS Nano 2010, 4, 5211-5216. 
1

2

3

4

5

6

7

8

9

10

11

12

13

14

15

16

17

18

19

20

21

22

23

24

25

26

27

28

29

30

31

32

33

34

35

36

37

38

39

40

41

42

43

44

45

46

47

48

49

50

51

52

53

54

55

56

57

58

59

60

(44) Beloborodov, I. S.; Lopatin, A. V.; Vinokur, V. M.; Efetov, K. B. Granular Electronic Systems. Rev. Mod. Phys. 2007, 79, 469-518.

\section{Table of content graphic}

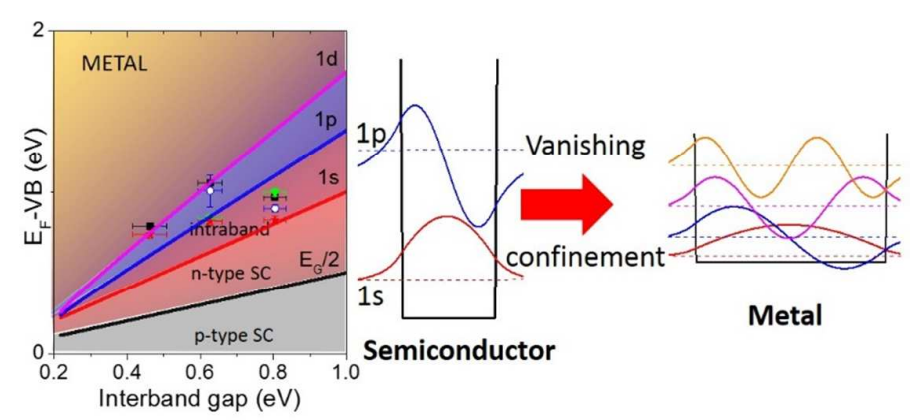

